\title{
Expediency of the Automated Perimetry Using the Goldmann V Stimulus Size in Visually Impaired Patients with Glaucoma
}

\author{
Adriana M. Morgan • Livia S. Mazzoli · Cristiano Caixeta-Umbelino • \\ Niro Kasahara (D)
}

Received: January 28, 2019 / Published online: March 13, 2019

(C) The Author(s) 2019

\section{ABSTRACT}

Introduction: White-on-white standard automated perimetry (AP) uses a white round stimulus with $0.43^{\circ}$ diameter and $4.0 \mathrm{~mm}^{2}$ area (Goldmann size III). Patients with low vision have difficulty seeing such a small stimulus and are often tested with perimetry using the size $\mathrm{V}$ stimulus with $1.72^{\circ}$ diameter and $64 \mathrm{~mm}^{2}$ area. We undertook an observational case-control study to compare the performance of patients on AP using two differently sized stimuli.

Methods: Patients with glaucoma and visual acuity worse than 20/100 underwent AP using the standard size III stimulus Swedish Interactive Threshold Algorithm (SITA) standard test and size $\mathrm{V}$ stimulus full threshold test. All

Enhanced Digital Features To view enhanced digital features for this article go to https://doi.org/10.6084/ m9.figshare.7770980.

Electronic supplementary material The online version of this article (https://doi.org/10.1007/s40123019-0175-9) contains supplementary material, which is available to authorized users.

A. M. Morgan - L. S. Mazzoli · C. Caixeta-Umbelino · N. Kasahara $(\square)$

Department of Ophthalmology, Irmandade da Santa Casa de Misericordia de Sao Paulo, São Paulo, SP, Brazil

e-mail: niro.kasahara@fcmsantacasasp.edu.br

N. Kasahara

Santa Casa de Sao Paulo School of Medical Sciences, São Paulo, SP, Brazil patients were familiar with the procedure, having done the test at least twice previously. Another group of glaucoma patients with visual acuity better than $20 / 40$ served as controls. The main outcome measures included test time, mean retinal sensitivity (MRS), foveal sensitivity (FS), fixation loss (FL), false positive (FP), false negative (FN), and the patient's subjective preference.

Results: Fifty patients were included in the study. Most preferred the size V stimulus target size test. For glaucoma patients, test time was shorter with size III; MRS and FS were higher with size $\mathrm{V}$; FL, FP, and FN did not differ between the tests.

Conclusion: AP with stimulus size V may be a good alternative to standard size III in selected visually debilitated patients who report difficulty undergoing a standard SITA 24-2 test.

Keywords: Automated perimetry; Glaucoma; Psychophysics; Stimulus size; Visual field

\section{INTRODUCTION}

Automated perimetry (AP) is the standard of care to detect and monitor glaucoma in clinical practice. White-on-white standard AP uses a white round stimulus with $0.43^{\circ}$ diameter and $4.0 \mathrm{~mm}^{2}$ area (Goldmann size III). Patients with low vision have difficulty seeing such small stimuli and are often tested using the 
Goldmann size V stimulus with $1.72^{\circ}$ diameter and $64 \mathrm{~mm}^{2}$ area. Although the use of stimulus $\mathrm{V}$ has been studied previously, none of these studies evaluated the patients' perspective or the reliability of this test strategy on clinical grounds [1-6]. The purpose of this study was to evaluate the performance of glaucoma patients with advanced disease during AP testing comparing two stimulus sizes. We hypothesize that patients with compromised vision would do better with AP testing with a larger stimulus size.

\section{METHODS}

This was an observational, cross-sectional, casecontrol study. All procedures performed in studies involving human participants were in accordance with the ethical standards of the institutional and/or national research committee and with the 1964 Helsinki Declaration and its later amendments or comparable ethical standards. The Santa Casa de Sao Paulo Ethics Committee in Human Research approved the study. Informed consent was obtained from all individual participants included in the study. The study group included patients with primary open-angle glaucoma and visual acuity worse than 20/100, and another group was comprised of glaucoma patients with visual acuity better than 20/40 who served as controls. All subjects underwent a complete eye examination including measurement of the best-corrected visual acuity (VA), slit-lamp biomicroscopy, tonometry with Goldmann applanation tonometry (Haag-Streit AG, Switzerland), gonioscopy with a Sussman four mirror handheld lens (Ocular Instruments, Bellevue, WA, USA), dilated ophthalmoscopy using a 78-D non-contact slit-lamp lens (Volk Optical Inc., Mentor, OH, USA), and a visual field test with HFV 750 (Carl Zeiss Meditec, San Leandro, CA, USA) using an appropriate lens to correct refractive errors. One eye of each patient had the visual field tested with two programs, the Swedish Interactive Threshold Algorithm (SITA) standard 24-2 test (size III stimulus) and size V stimulus full 24-2 threshold test, FASTPAC strategy. The order of which test was done first was randomly assigned. Both examinations were done on the same day after a short period of rest. All patients were familiar with the procedure, having done the test at least twice previously. The AP testing and all examinations were done by one of us (AMM).

The main outcome measures were the test time, mean retinal sensitivity (MRS), foveal sensitivity (FS), fixation loss (FL), false positive (FP), false negative (FN), and the patient's subjective preference. MRS was calculated as the average of the measured threshold retinal sensitivity value for each of the 54 test points in the numeric plot. Data between the groups and between stimulus sizes within the groups were compared using Students' $t$ test for continuous variables and the $\chi^{2}$ test on a contingency table for categorical variables. $P$ values less than 0.05 were deemed statistically significant.

\section{RESULTS}

Fifty patients were included in the study. Table 1 displays the demographic characteristics and AP performance between the groups. Most patients preferred the size $\mathrm{V}$ stimulus target size test. Comparison of the two stimulus size tests for patients with low vision is shown in Table 2 . Test time was shorter with size III; MRS and FS were higher with size $\mathrm{V}$, and the catch trials (FL, FP, and FN) did not differ between the tests. Table S3 in the electronic supplementary material displays the comparison of the two stimulus size tests for the control group. Figure 1(a) shows the printout of the SITA 24-2 test and 1(b) displays the test result of the size $\mathrm{V}$ stimulus full threshold. In this patient, the retinal sensitivity was higher and the falsenegative rate was lower with stimulus size $\mathrm{V}$.

\section{DISCUSSION}

The results of this study favor the use of a size $\mathrm{V}$ stimulus in some glaucoma patients with advanced disease. Although the catch trials did not differ between the two stimulus size tests, most patients preferred the AP test with the size $\mathrm{V}$ target. This fact, however, does not 
Table 1 Demographic features and automated perimetry with stimulus size III test results between groups

\begin{tabular}{llll}
\hline & Study group & Control group & P value \\
\hline Age (years) & $61.7 \pm 13.2$ & $64.7 \pm 10.1$ & 0.532 \\
Gender (M:F) & $12: 16$ & $10: 12$ & 0.679 \\
Ethnicity & & & 0.534 \\
White & 18 & 14 & \\
Non-white & 10 & 08 & \\
VA (logMAR) & $0.97 \pm 0.26$ & $0.19 \pm 0.18$ & 0.000 \\
Test duration (min) & $8.0 \pm 1.6$ & $7.2 \pm 1.8$ & 0.320 \\
FS & $21.8 \pm 8.6$ & $28.3 \pm 7.5$ & 0.060 \\
MRS & $15.1 \pm 10.6$ & $24.3 \pm 7.4$ & 0.020 \\
FL & $0.2 \pm 0.2$ & $0.1 \pm 0.4$ & 0.789 \\
VFI (\%) & $39.4 \pm 37.5$ & $76.6 \pm 29.3$ & 0.031 \\
MD & $-21.3 \pm 9.7$ & $-11.2 \pm 9.1$ & 0.016 \\
PSD & $4.5 \pm 2.2$ & $5.7 \pm 3.7$ & 0.367 \\
FP & $0.0 \pm 0.0$ & $0.0 \pm 0.0$ & 0.115 \\
FN & $0.2 \pm 0.3$ & $0.0 \pm 0.0$ & 0.030 \\
Patient preference for size V (\%) & 71.4 & 70.0 & 0.941 \\
\hline
\end{tabular}

min minutes, $M$ male, $F$ female, $M R S$ mean retinal sensitivity, $F S$ foveal sensitivity, $V F I$ visual field index, $M D$ mean deviation, $P S D$ pattern standard deviation, $F L$ fixation loss, $F P$ false positive, $F N$ false negative

Table 2 Automated perimetry test results comparing stimulus size III and V for the study group

\begin{tabular}{lccc}
\hline & Size III & Size $\mathbf{~}$ & $\boldsymbol{P}$ value \\
\hline Test duration (min) & $6.8 \pm 0.8$ & $8.0 \pm 1.6$ & 0.046 \\
FS & $16.2 \pm 9.4$ & $21.8 \pm 8.6$ & 0.009 \\
MRS & $9.3 \pm 8.7$ & $15.1 \pm 10.6$ & 0.000 \\
FL & $0.3 \pm 0.4$ & $0.2 \pm 0.2$ & 0.209 \\
FP & $0.0 \pm 0.0$ & $0.0 \pm 0.0$ & 0.358 \\
FN & $0.09 \pm 0.08$ & $0.2 \pm 0.4$ & 0.251 \\
\hline
\end{tabular}

min minutes, $M R S$ mean retinal sensitivity, $F S$ foveal sensitivity, $F L$ fixation loss, $F P$ false positive, $F N$ false negative

necessarily mean that AP with size $\mathrm{V}$ stimulus is better than size III. A larger stimulus target would be more easily perceived than a smaller one, so that one could expect the $\mathrm{FN}$ rateretesting a previously tested location with a stimulus brighter than the measured threshold value-to be lower. Besides, the use of size $\mathrm{V}$ stimulus reduces variability in moderately damaged and normal sensitivity test locations in subjects with glaucoma [1]. In our study, however, the FN rates were low for both size III and $\mathrm{V}$ tests. A possible explanation for this observation is that all subjects included in the study were experienced with AP, having 
a Monitor de Fixacão: Fixacão/Ponto Cego Estímulo: III. Branco Alvo de Fixacão: Central

Perdas de Fixacão: 16/19 xx

Erros Falsos POS: $2 \%$

Erros Falsos NEG: $20 \%$

Duracão do Teste: 07:21

Fóvea: $31 \mathrm{~dB} \%$
Fundo: 315 Ast

Estratégia: SITA-Standard
Diâmetro da Pupila: $4.8 \mathrm{~mm}$

Acuidade Visual:

$R X:+3.25$ DS $\quad D C X$
Data: 06-10-2017

Horas: 10:00 AM

Idade: 62

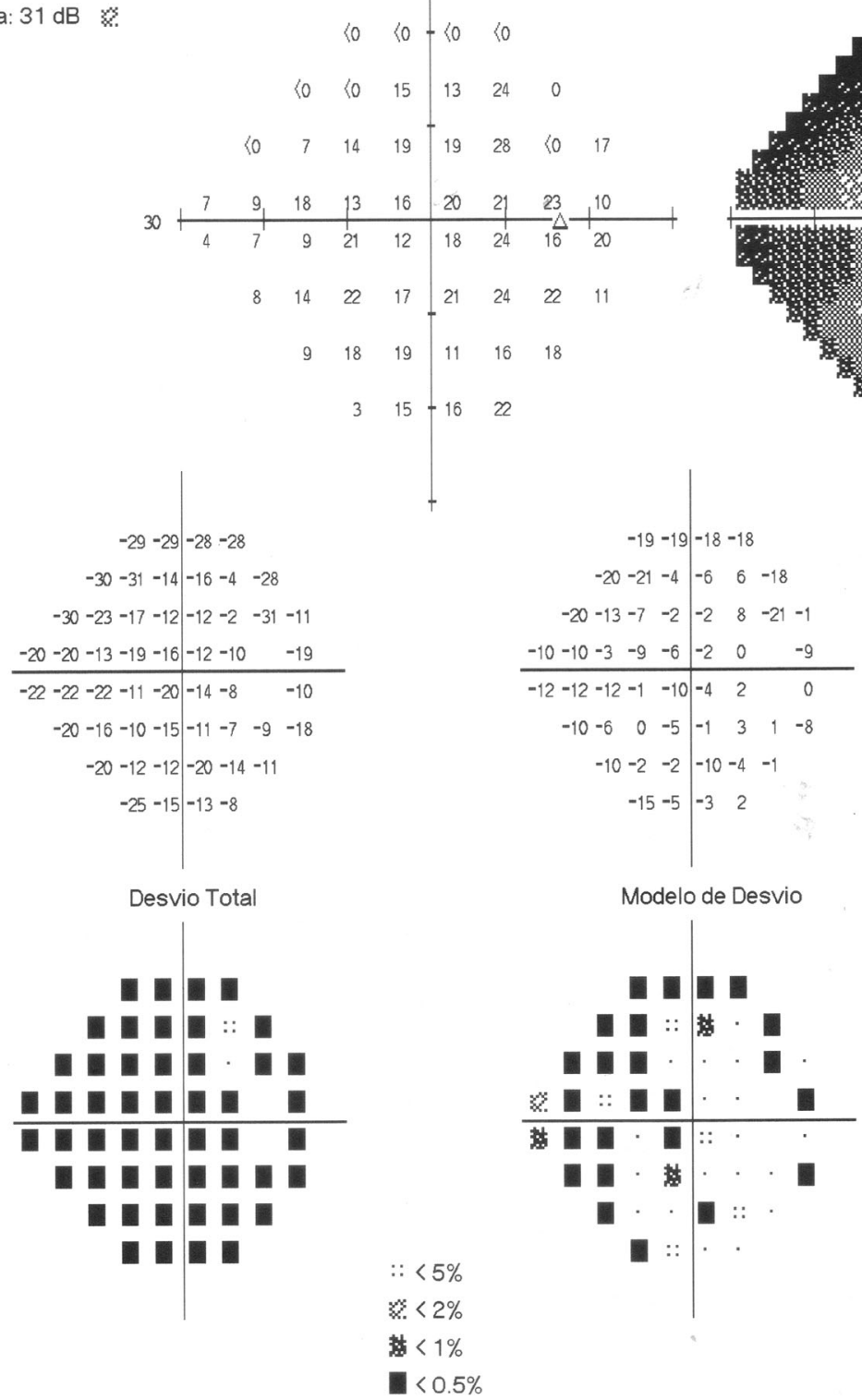

Fig. 1 a Printout of the SITA 24-2 test result using the regular stimulus size III. b Printout of the full threshold FASTPAC with stimulus size $\mathrm{V}$

undergone the examination previously. The SITA standard test does not compute the FN rate in patients with very advanced disease and low $\mathrm{MD}$ values, so that the comparison of $\mathrm{FN}$ rates between the two stimuli size tests was hindered. Besides, in glaucoma patients with advanced disease, the FN rate may be explained by the increased variability in the threshold values 


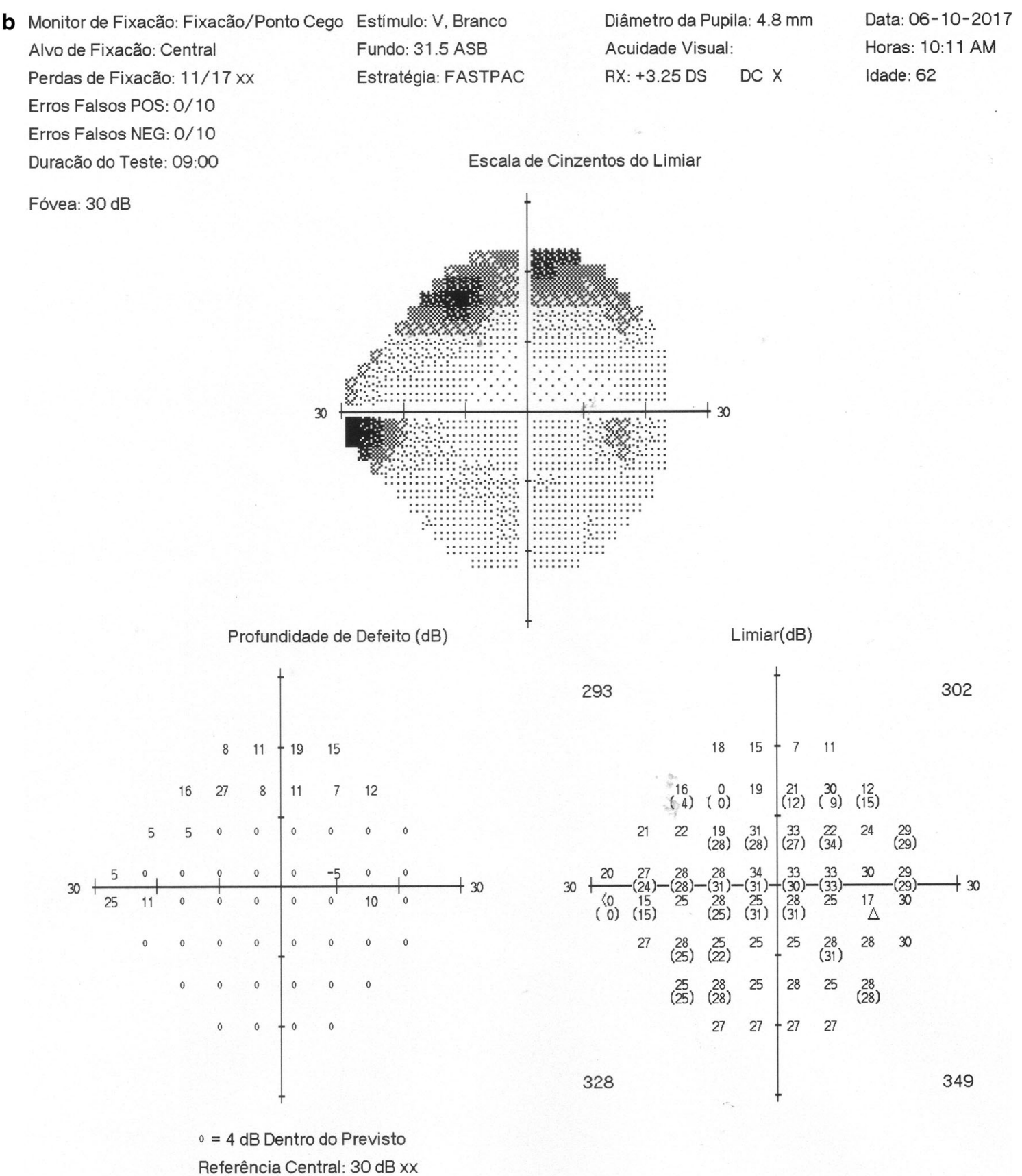

Fig. 1 continued

typically found in such eyes and therefore represents eye status and might not be indicative of attention [7].

In this study, test duration was shorter with the SITA standard strategy. Both SITA standard and FASTPAC are strategies aimed to reduce examination time. FASTPAC estimates the threshold from a single reversal of the patient's response in the staircase procedure using a fixed step size of $3 \mathrm{~dB}$ [8]. In the SITA standard strategy, threshold values and measurement errors of threshold values are continuously estimated during the test using maximum posterior probability calculations in visual field models 
[9]. The average 1 min longer duration, however, does not seem to be clinically significant.

Values of retinal sensitivity were $6 \mathrm{~dB}$ higher when tested with stimulus V. Previous studies reported an increase up to $7.6 \mathrm{~dB}$ in the mean retinal sensitivity for both glaucoma and nonglaucoma subjects using the Octopus perimeter $[10,11]$. This can be explained by the relation between stimulus size and luminance. There is a fixed ratio between the size of a stimulus and its intensity when plotting isopters. For any given isopter, a change in either stimulus size or intensity will plot the same isopter if the other is varied such that the ratio between the size and intensity is kept constant. A fourfold increase in the stimulus size would plot the same isopter if the spot luminance was decreased by a factor of 3.16 [12].

Swanson et al. evaluated the effect of stimulus size on sensitivity of patients with retinitis pigmentosa. AP (full threshold programs) was performed using stimulus sizes III and V. The authors concluded that, in the damaged regions of the visual field, an increase in stimulus size from III to V could produce abnormally large increases in perimetric sensitivity. Whereas size III may be more useful for detection of visual field abnormality, size $\mathrm{V}$ would be more useful for monitoring progression of advanced disease [13]. The authors, however, did not compare test time and reliability indexes between the two strategies.

One limitation of using size $\mathrm{V}$ stimulus in AP is the lack of normative database. In the defect depth printout, the perimeter uses a mathematical model to predict the normal retinal sensitivity for each test point by deriving a slope value per degree of eccentricity from the fixation. Assessment of visual function progression with stimulus size $\mathrm{V}$ is another limitation. There is no software like the Humphrey Guided Progression Analysis $\left(\mathrm{GPA}^{\mathrm{TM}}\right.$ ) to evaluate visual field progression for stimulus $\mathrm{V}$. Besides, we used $20 / 100$ and 20/40 as an arbitrary cutoff for VA for the study and control groups, respectively, to have a better separation between the groups. Anecdotal reports suggest that standard AP can be done in patients with up to 20/200 vision.

Given all the aforementioned limitations, how can AP with size $\mathrm{V}$ stimulus size be incorporated in clinical practice? It is not uncommon to find patients complaining about the AP test. Maybe for these patients AP with stimulus $\mathrm{V}$ could be offered instead as an alternative. For glaucoma suspects with compromised vision, for whatever reason, the size $\mathrm{V}$ stimulus test could be performed initially to obtain an indication of the functional status and disease detection. Later, in subsequent testing, standard size III could be used to evaluate functional progression. For patients with low vision and needing a specific optical visual aid, AP with size V stimulus could be used to evaluate visual function.

\section{CONCLUSIONS}

Since AP is a subjective examination dependent on the subject's attention, any adjustment to increase patient cooperation is a valid option to help provide more dependable test results to detect and monitor glaucoma in visually debilitated patients. AP with stimulus size $\mathrm{V}$ may be a good alternative to standard size III in selected visually debilitated patients who report difficulty undergoing the conventional SITA 24-2 test.

\section{ACKNOWLEDGMENTS}

We thank the participants of the study.

Funding. No funding or sponsorship was received for this study or publication of this article. The article processing charges were funded by the authors.

Authorship. All named authors meet the International Committee of Medical Journal Editors (ICMJE) criteria for authorship for this article, take responsibility for the integrity of the work as a whole, and have given their approval for this version to be published.

Author Contributions. Concept and design: NK, CCU; data acquisition: AMM, LSM; data analysis/interpretation: AMM, NK; drafting manuscript: AMM, NK; critical revision of 
manuscript: LSM, CCU, AMM, NK; supervision: CCU, NK.

Prior Presentation. Presented in part at the Association for Research in Vision and Ophthalmology Annual Meeting, April 29-May 3, 2018, Honolulu, Hawaii.

Disclosures. Adriana M. Morgan, Livia S. Mazzoli, Cristiano Caixeta-Umbelino, and Niro Kasahara have nothing to disclose.

Compliance with Ethics Guidelines. All procedures performed in studies involving human participants were in accordance with the ethical standards of the institutional and/or national research committee and with the 1964 Helsinki Declaration and its later amendments or comparable ethical standards. The Santa Casa de Sao Paulo Ethics Committee in Human Research approved the study. Informed consent was obtained from all individual participants included in the study.

Data Availability. The data sets during and/ or analysed during the current study are available from the corresponding author on reasonable request.

Open Access. This article is distributed under the terms of the Creative Commons Attribution-NonCommercial 4.0 International License (http://creativecommons.org/licenses/ by-nc/4.0/), which permits any noncommercial use, distribution, and reproduction in any medium, provided you give appropriate credit to the original author(s) and the source, provide a link to the Creative Commons license, and indicate if changes were made.

\section{REFERENCES}

1. Wall M, Kutzko KE, Chauhan CBC. Variability in patients with glaucomatous visual field damage is reduced using size V stimuli. Invest Ophthalmol Vis Sci. 1997; 38: 426-35.
2. Wall M, Brito CF, Woodward KR, Doyle CK, Kardon $\mathrm{RH}$, Johnson CA. Total deviation probability plots for stimulus size $\mathrm{V}$ perimetry. A comparison with size III stimuli. Arch Ophthalmol. 2008; 126: 473-9.

3. Wall M, Doyle CK, Eden T, Zamba KD, Johnson CA. Size threshold perimetry performs as well as conventional automated perimetry with stimulus sizes III, V, and VI for glaucomatous loss. Invest Ophthalmol Vis Sci. 2013;54:3975-83.

4. Gardiner SK, Demirel S, Goren D, Mansberger SL, Swanson WH. The effect of stimulus size on the reliable stimulus range of perimetry. Tran Vis Sci Tech. 2015;4:10.

5. Phu J, Khuu SK, Zangerl B, Kalloniatis M. A comparison of Goldmann III, V and spatially equated test stimuli in visual field testing: the importance of complete and partial spatial summation. Ophthalmic Physiol Opt. 2017;37:160-76.

6. Wall M, Zamba GKD, Artes PH. The effective dynamic ranges for glaucomatous visual field progression with standard automated perimetry and stimulus sizes III and V. Invest Ophthalmol Vis Sci. 2018;59:439-45.

7. Bengtsson B, Heijl A. False-negative responses in glaucoma perimetry: indicators of patient performance or test reliability? Invest Ophthalmol Vis Sci. 2000;41:2201-4.

8. Schaumberger M, Schafer B, Lachenmayr BJ. Glaucomatous visual fields. fastpac versus full tthreshold strategy of the humphrey field analyzer. Invest Ophthalmol Vis Sci. 1995; 36: 1390-7.

9. Bengtsson B, Olsson J, Heijl A, Rootzén H. A new generation of algorithms for computerized threshold perimetry, SITA. Acta Ophthalmol. 1997;75:368-75.

10. Choplin NT, Sherwood MB, Spaeth GL. The effect of stimulus size on the measured threshold values in automated perimetry. Ophthalmol. 1990;97:371-4.

11. Zulauf M, Caprioli J. Indications for stimulus 3 and 5 in automatic perimetry. Prelim Results Klin Monbl Augenheilkd. 1994;204:407-8.

12. Sloan LL. Area and luminance of test object as variables in examination of the visual field by projection perimetry. Vis Res. 1961;1:121-38.

13. Swanson WH, Felius J, Birch DG. Effect of stimulus size on static visual fields in patients with retinitis pigmentosa. Ophthalmol. 2000;107:1950-4. 